\title{
La théorie cognitive des passions chez Chrysippe
}

Une opinion faible peut-elle se traduire par une horme pleonazousa?

\section{Inmaculada Hoyos Sanchez}

\section{(2) OpenEdition}

Journals

Édition électronique

URL : https://journals.openedition.org/philosant/627

DOI : $10.4000 /$ philosant.627

ISSN : 2648-2789

Éditeur

Éditions Vrin

\section{Édition imprimée}

Date de publication : 1 novembre 2016

Pagination : 153-180

ISBN : 978-2-7574-1472-9

ISSN : 1634-4561

Référence électronique

Inmaculada Hoyos Sanchez, "La théorie cognitive des passions chez Chrysippe », Philosophie antique [En ligne], 16 | 2016, mis en ligne le 01 novembre 2018, consulté le 02 décembre 2022. URL : http:// journals.openedition.org/philosant/627 ; DOI : https://doi.org/10.4000/philosant.627

\section{(c)}

Creative Commons - Attribution - Pas d'Utilisation Commerciale - Pas de Modification 4.0 International - CC BY-NC-ND 4.0

https://creativecommons.org/licenses/by-nc-nd/4.0/ 


\section{LA THÉORIE COGNITIVE DES PASSIONS CHEZ CHRYSIPPE Une opinion faible peut-elle se traduire par une horme pleonazousa? Inmaculada Hoyos SÁNCHEZ Université de Grenade/Centre Léon Robin}

RÉSUMÉ. Cet article propose une analyse de l'un des principaux problèmes de la théorie cognitive des passions chez Chrysippe, à savoir, comment expliquer que le jugement faux et faible, qui définit une passion, se traduise par une horme forte et violente. Le cas de Médée, paradigme de cette difficulté, est particulièrement intéressant. La solution de Posidonius affirme que les mouvements affectifs (pathetikai kineseis), qui impliquent, d'après Galien, une partie irrationnelle de l'âme, sont la cause de l'impulsion débridée. Néanmoins, mon intention est de développer la thèse de la prépondérance des croyances (Nussbaum, Graver) afin de montrer que la théorie cognitive de Chrysippe est suffisamment flexible pour expliquer le cas de Médée en termes de croyances, et qu'il n'est pas nécessaire de faire appel à une autre partie irrationnelle de l'âme pour résoudre le problème posé. L'idée principale est qu'une croyance peut être faible et fausse mais qu'elle peut acquérir une place prépondérante sur d'autres (y compris celles qui sont vraies) et, par conséquent, avoir un pouvoir causal plus important.

SUMMARY. The core aim of this paper is to analyse one of the main problems of Chrysippus's cognitive theory of passion, that is, how to explain that the false judgement, and therefore a weak one, which defines a passion, turns into a strong and violent horme. The case of Medea, which is paradigmatic of this difficulty, receives special attention. Posidonius's solution shows that the affective movements (pathetikai kineseis), which involve, according to Galen, another irrational part of the soul, are the cause of excessive impulse. Nevertheless, my intention is to develop the theory of the salience of beliefs (Nussbaum, Graver) to show that Chrysippus's cognitive theory is flexible enough to explain Medea's case in terms of beliefs and that it is not necessary to resort to an irrational part of the soul to solve the problem posed. The main idea is that a belief can be weak and false but it can acquire a prominent place regarding the other beliefs (even when they are true) and, in that sense, have a greater causal power.

Philosophie antique, $\mathrm{n}^{\circ} 16$ (2016), 153-180 

Le but principal de cet article* est de présenter et essayer d'atténuer l'une des plus grandes difficultés de la théorie cognitive des passions chez Chrysippe. La problématique est la suivante : selon Chrysippe, la passion (pathos) est un faux jugement de valeur ${ }^{1}$. Mais le faux jugement à l'œuvre dans la passion n'est pas suffisant pour décrire ce en quoi consiste la passion, puisque la passion n'est pas seulement une erreur cognitive. La passion repose sur une erreur morale, et, de plus, elle a pour conséquence un débordement, celui de l'horme pleonazousa. Or la passion est une opinion (doxa), et de ce fait, elle comporte un assentiment faible, différent de la fermeté de la katalepsis ${ }^{2}$.

* La recherche qui a permis la rédaction de cet article a été réalisée lors d'un séjour postdoctoral, financé par l'Université de Grenade, au Centre Léon Robin de recherches sur la pensée antique. Je tiens à exprimer ma très chaleureuse reconnaissance à tous ses membres, et notamment à son directeur, Jean-Baptiste Gourinat, pour son aide précieuse qui m'a permis d'approfondir mes connaissances sur le stoïcisme ainsi que pour la relecture attentive de ce texte, ses remarques enrichissantes et ses suggestions précises en ce qui concerne la traduction française des textes de Galien (et pour avoir gentiment vérifié mon français). Une première version de cet article a été présentée à l'Université Ca'Foscari de Venise lors d'un Colloque sur Anima, corpo, passioni nel pensiero antico (4-5 octobre 2013). Je remercie les organisateurs, Carlo Natali et Cristina Viano, pour leur invitation, ainsi que les participants pour leurs questions stimulantes.

1. «Car l'appétit, la colère, la peur et toutes les choses semblables sont des opinions et des jugements corrompus ». Plutarque, De la vertu morale, 446F-447A = SVF III $459=$ Long \& Sedley (LS) 65G. Je cite la traduction française de Brunschwig \& Pellegrin 2001.

2. Les définitions de l'opinion comme assentiment faible sont nombreuses depuis Zénon. Stobée souligne « qu'ils [les stoïciens] disent que le sage ne fait jamais de fausse supposition, et qu'il ne donne absolument aucun assentiment à quoi que ce soit d'incognitif, du fait qu'il n'a pas d'opinions et qu'il n' ignore rien. Car l'ignorance est un assentiment instable et faible (hupolepsis asthenes) 》 (Stobée II, 111, 18-112, $8=$ SVF III, 548 extrait partiel = LS 41G). Voir aussi Stobée II, 88, 22-89, 3 = SVF III, 378 extrait partiel = LS 65C : « Dans le cas de toutes les passions de l'âme, quand [les stö̈ciens] les appellent des "opinions”, "opinion” est utilisé à la place de "supposition faible", et "nouvelle" à la place de "stimulus d'une contraction ou d'un gonflement irrationnels” ». Et aussi, Sextus Empiricus, Adversus mathematicos, VII 151 = SVF I 67, où nous pouvons lire que l'opinion est un assentiment faible et faux. 
Mais la passion est forte et violente, elle n'est pas faible ${ }^{3}$. Comment expliquer qu'un jugement faible se traduise par une horme forte et violente ? La théorie cognitive peut expliquer, par exemple, le caractère volatil et changeant de la passion. Mais cette théorie a de grandes difficultés à expliquer ce qui est le plus caractéristique de la passion : sa démesure, son emportement, sa violence. Poser l'existence d'une partie irrationnelle de l'âme, telle que l'affirmaient les platoniciens, permettait d'expliquer de manière plus satisfaisante ce caractère particulier de la passion. Nous pouvons ainsi penser, comme Galien, que d'autres stoïciens, dont la psychologie se rapproche plus de celle des platoniciens, à savoir Posidonius, sont mieux à même de résoudre ce problème.

Mon but est donc ici de déterminer si la théorie cognitive de Chrysippe est suffisamment riche pour résoudre cette difficulté, ou s'il est nécessaire de faire appel à d'autres facteurs irrationnels pour expliquer les causes des passions, et s'il faut, par conséquent, renoncer à cette théorie. Cette dernière question est liée au débat sur l'interprétation de ces facteurs irrationnels : faut-il considérer, comme le veut Galien, qu'ils impliquent l'existence d'une partie irrationnelle de l'âme différente et opposée à la partie rationnelle ? Galien a-t-il raison dans son interprétation de Chrysippe et de Posidonius ? Si tel est le cas, la théorie cognitive des passions de Chrysippe doit être abandonnée.

Nombreux sont les interprètes qui ont posé cette question de façon claire et féconde : Bénatouil, Gill, Gourinat, Joyce, Graver, Sorabji, Tieleman, etc. Pour ma part, je partage la thèse de la théorie cognitive des passions, que l'on peut tirer de la philosophie de Chrysippe. Mon intention est donc de partir des contributions de ces interprètes et d'y ajouter de nouveaux arguments tirés de la lecture de certains textes de Posidonius et Chrysippe afin de répondre à la question principale exposée ici, à savoir, si une opinion faible peut devenir une passion forte ou, en d'autres termes, si elle peut se traduire par une horme pleonazousa.

3. La problématique est très bien posée chez Stobée et Arius Didyme : « "Irrationnel”

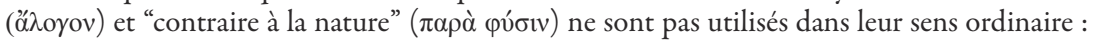

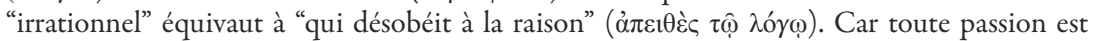
excessivement puissante, puisque les gens en état de passion voient fréquemment qu'il n'est pas convenable de faire ceci ou cela, mais sont entraînés par l'intensité ( $\beta 1 \alpha \sigma \tau 1 \kappa o ́ v)$ de la

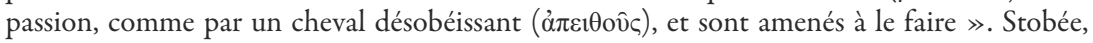
Eclogae, II, 89, 4 Wachsmuth = LS 65A. Chez Arius Didyme nous pouvons juste après lire : «Par conséquent, souvent les gens avouent même ceci, en prononçant cette phrase couram-

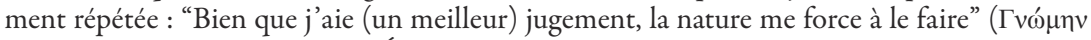

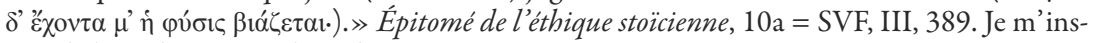
pire de la traduction anglaise de Pomeroy 1999. 


\section{Posidonius vs. Chrysippe : les textes}

Les textes qui me semblent les plus pertinents pour résoudre la difficulté mentionnée font partie de l'ouvrage de Galien, Les doctrines d' Hippocrate et de Platon. Dans le premier texte Galien affirme :

Mais Posidonius était en désaccord complet avec ces deux opinions. Il ne considère les passions $(\pi \alpha ́ \theta \eta)$ ni comme des jugements ni comme des résul-

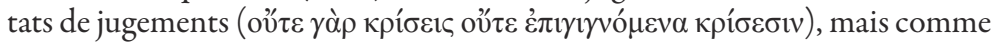

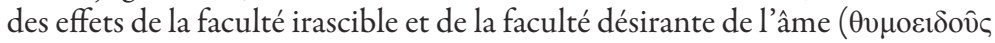

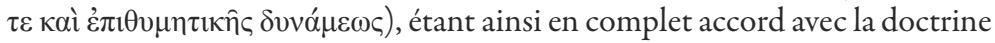
ancienne. Dans son étude Despassions, il demande souvent aux chrysippéens : «Quelle est la cause de l'impulsion excessive ? » ( $\tau$ íc $\dot{\eta} \tau \hat{\eta} \varsigma \pi \lambda \varepsilon o v \alpha \zeta o v ́ \sigma n \varsigma$

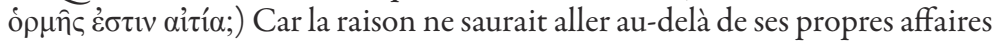
et de ses propres mesures. Ainsi est-il évident qu'une autre faculté, irration-

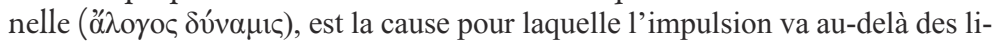
mites de la raison, comme la cause qui fait que la course va au-delà des limites

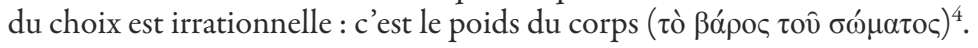

D'après Galien, Posidonius s'écarte de la définition de la passion que Zénon défend ainsi que de celle de Chrysippe et, donc, il considère que la raison ne peut pas être la source de la passion. C'est parce que la cause de l'impulsion excessive qu'est la passion ne peut se trouver dans la raison: la raison ne peut pas se surpasser elle-même. La raison est ordre et mesure, elle ne peut donc pas produire de désordre. C'est pourquoi il existe une autre partie dans l'âme, qui serait irrationnelle, et qui explique la cause de l'impulsion excessive.

Le deuxième texte qui évoque ce même problème fait partie du livre IV de l'ouvrage de Galien cité précédemment. Ce texte me semble remarquable car il permet de mettre en relation la difficulté mentionnée avec un autre cas, celui de Médée, qui est notamment lié à l'objection posée. Dans ce texte, Posidonius souligne l'impasse dans laquelle se trouve la théorie de Chrysippe. Dans la réflexion de Chrysippe sur les passions, une sorte de cercle vicieux se produit, lequel nous empêche d'avancer dans la recherche sur la cause des passions.

Posidonius écrit ensuite : « Non seulement, d'après lui [Chrysippe], la raison est détournée dans les désirs passionnels, mais il nous fait de surcroît estimer que, même si l'objet de notre désir ne présente pas d'intérêt ( $\mu$ ทे

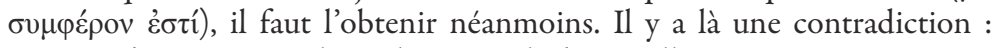
nous tendrions vers quelque chose avec l'idée qu'elle présente un important intérêt ; et, en raison de son importance, même si elle va contre notre intérêt, nous estimerions que, même ne présentant aucun avantage, bien au contraire,

4. Galien, De Placitis Hippocratis et Platonis (PHP), IV 3, 3-5, p. 248-249 De Lacy = Posidonius, Fr. 157 Edelstein \& Kidd (EK) = LS 65K (trad. Brunschwig \& Pellegrin 2001 à peine modifiée. Ils traduisent par «faculté ardente » au lieu de «faculté irascible »). 


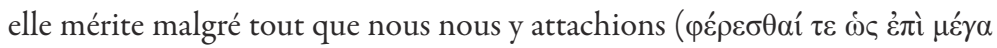

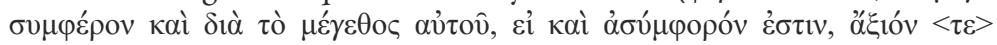

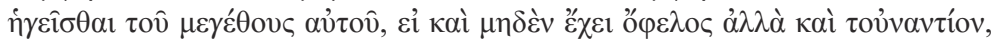

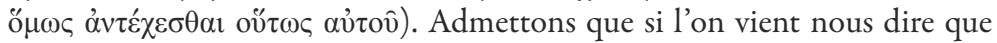
nous n'y avons aucun intérêt, nous jugions que c'est là déraisonner, et si l'on prétend nous démontrer que cela va au contraire contre notre intérêt, nous jugions que ce sont là des radotages car ce que nous poursuivons présente un grand avantage. Reste cette invraisemblance : parce qu'on estime que c'est un grand bien, on pense qu' il faut, même si c'est un grand mal, l'obtenir quand même, et l'on clame :

\section{Laisse-moi périr ; c'est là ce qui m'importe maintenant.}

Il n'est pas vraisemblable en effet que la cause de cette attitude réside dans l'opinion de la présence d'un grand bien vers lequel on tend. Cette cause

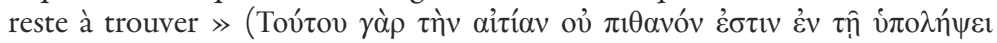

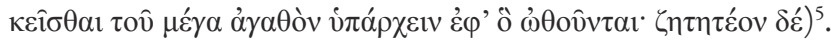

J'ai choisi ce texte car je pense qu'il permet de rattacher l'objection de Posidonius à l'exemple de Médée. Médée pense que la vengeance est l'action appropriée pour répondre à l'offense de Jason. Mais elle estime que se venger en tuant ses enfants est nocif. Nous pourrions donc affirmer que lobjet de son désir va contre son intérêt. Néanmoins, elle les tue. Ainsi, ce n'est pas l'appréciation de l'objet de son action qui explique le passage à l'acte, puisqu'un jugement positif (estimer l'action bonne) devrait la conduire à agir et un jugement négatif (l'estimer nocive) à ne pas agir. L'explication de Chrysippe n'explique donc rien. S'appuyer sur les opinions de Médée à l'égard de l'objet, bon ou nocif, ne suffit pas pour éclairer la cause des passions. Telle est la critique générale de fond.

La réponse de Posidonius, selon la lecture de Galien, consiste à affirmer qu'il existe une autre partie, irrationnelle, de l'âme qui explique le comportement de Médée et l'excès de l'impulsion. Il existe une poussée émotionnelle (pathetike holke), un mouvement de la capacité émotionnelle (to pathetikon), qui, d'après Galien, est différent de la raison et qui est la cause de l'excès de l'impulsion ${ }^{6}$. L'erreur pratique qu'est la passion est différente de l'erreur

5. Galien, PHP IV, 5, 42-45, p. 268-271 De Lacy = Posidonius Fr. 164 (6) EK. (trad. Fillion-Lahille 1984, p. 145 à peine modifiée).

6. Cf. Galien, PHP V, 5, 21 = Posidonius, Fr. $169 \mathrm{EK}$; et Galien, $P H P$ IV, 7, $33=$ Posidonius, Fr. 165 EK. Il existe une troisième occurrence de l'expression pathetikai kineseis chez Galien, PHP IV, 7, 37 = Posidonius, Fr. 165 EK. En ce qui concerne le débat pour savoir si cette expression de Posidonius, pathetikai kineseis, fait référence à une partie de l'âme qui est différente de la partie rationnelle, Kidd 1971, p. 203, avance que Posidonius rejette le terme «parties » (mere) de l'âme et donc, la théorie de la séparation locale. Aussi, Posidonius rejette-t-il le terme eide, et il insiste simplement sur les différentes capacités (dunameis) qui ne sont pas localisées de manière spécifique. Posidonius maintient également que l'âme est une substance unitaire simple. Mais il rejette l'argument de Chrysippe, selon lequel une substance simple a besoin d'une unique ou d'une simple capacité. Gourinat 1996, p. 27, note que 
purement cognitive dont la cause est l'ignorance. Selon Galien, la cause de l'erreur pratique réside dans une partie irrationnelle de l'âme. Posidonius soutient-il effectivement la position qui lui est attribuée par Galien ? La lecture que fait Galien de Chrysippe et Posidonius est-elle correcte ?

\section{La théorie cognitive prolongée : Chrysippe et ses élaborations contemporaines (Tieleman et Graver)}

Tieleman et Graver sont deux des interprètes qui ont répondu au problème qui se pose dans ces deux textes à partir de la théorie cognitive. Dans son ouvrage, Tieleman fait un effort important pour sauver Chrysippe des accusations de Galien et pour rapprocher les théories de Chrysippe et de Posidonius. L'argument général qu'il présente consiste à montrer que l'aspect cognitif (Chrysippe) et l'aspect physique (Posidonius) sont deux aspects de la même chose, c'est-à-dire d'une passion, compte tenu de la psychologie moniste et matérialiste du stoïcisme $\mathrm{p}^{7}$. La poussée émotionnelle que, selon Kidd, Posidonius introduit pour expliquer la cause de l'impulsion excessive, est une autre façon de faire référence à la « condition supplémentaire mal connue » dont Chrysippe parle et qui fait référence à la nature corporelle de l'âme ${ }^{8}$. Dans notre premier texte, l'expression « $\sigma \omega ́ \mu \alpha \tau o \varsigma$ », qui est introduite pour expliquer la cause de l'impulsion excessive, est un aspect non rationnel que la théorie cognitive reconnaît mais, d'après Tieleman, il s'agit d'un aspect qui correspond à l'opinion ou au jugement. Le tonos ou la tension physique de l'âme, dont Chrysippe parle dans d'autres textes, ainsi que les opinions, sont deux aspects de la même chose, c'est-à-dire une passion'. Tieleman reconnaît qu'il existe des aspects non rationnels (il préfère ce terme à celui d'《irrationnel ») dans l'analyse causale des passions chez Chrysippe. Cependant, ces facteurs non rationnels sont aussi inclus dans la théorie cognitive. Mais cela ne veut pas dire que Galien ait raison quand il interprète ces aspects non rationnels comme des

Posidonius préfère parler d'une faculté rationnelle et d'une autre passionnelle, plutôt que d'utiliser le terme « irrationnelle ». Il semble que Posidonius pense que l'âme a deux facultés contraires qui sont caractérisées dans un sens platonicien, sans penser, à première vue, aux quatre facultés de Chrysippe. Mais, selon Gourinat, il est essentiel que Posidonius ne considère pas ces facultés comme des parties de l'âme et que Posidonius ne leur assigne pas une localisation séparée, mais une seule et même substance. Fillion-Lahille dit aussi que le siège de la passion coïncide avec le siège de la raison (Fillion-Lahille 1984, p. 154, avec référence aux Epistulae morales, 23). Dans la même ligne d'interprétation, Gill 1998 soutient que l'allusion aux mouvements affectifs n'implique pas l'existence des différentes parties, comme le veut Galien. En conclusion, il me semble qu'il existe suffisamment d'arguments pour penser que l'expression pathetikai kineseis ne fait pas référence à une partie de l'âme différente de la rationnelle.

7. Ioppolo 1995, p. 54, soutient également cette thèse.

8. Cf. Tieleman 2003, p. 260-263.

9. Ibid., p. 250-252. Cf. Galien, PHP, IV, 6, 1-12. 
pouvoirs irrationnels situés dans une partie irrationnelle de l'âme différente de la partie rationnelle. Posidonius et Chrysippe, lorsqu'ils reconnaissent l'existence de facteurs non rationnels, ne s'engagent pas dans la psychologie tripartite platonicienne. Selon Tieleman, Galien s'est trompé.

Quant au deuxième texte, certains de ses aspects peuvent être expliqués par ce que Tieleman et d'autres interprètes appellent la « théorie de la double structure cognitive de l'émotion », qui est déjà présente chez Chrysippe et qui peut être reconstruite à partir du témoignage de Cicéron dans ses Tusculanes ${ }^{10}$. Tieleman montre que Chrysippe distingue deux jugements dans son analyse des passions, c'est-à-dire un jugement de type 1 , à savoir que « $\mathrm{X}$ est quelque chose de bon ou de mauvais »; et un jugement de type 2 sur la réponse appropriée à cet évènement. Ce que je veux donc faire ressortir, c'est que Tieleman avance que Chrysippe introduit un facteur quantitatif pour expliquer le passage du jugement de type 1 au jugement de type 2. C'est la grandeur du bien apparent qui conduit à ce deuxième jugement. Si nous considérons qu'une femme n'est pas seulement quelque chose de bien, mais la meilleure chose qui existe (jugement type 1), il semble alors normal d'être emporté avec passion lorsqu'elle se présente, et de refuser toute raison pour agir d'une autre façon (jugement type 2 ) $^{11}$. Cette lecture de Tieleman me semble intéressante car elle permet d'atténuer le problème abordé ici. La véhémence de l'impulsion de la passion peut être expliquée par la croyance concernant la grande valeur de l'objet en question. Il est vrai que cette croyance est une opinion fausse, et qu'une opinion fausse, selon les stö̈ciens, ne peut pas entraîner un assentiment fort, comme le montrent les définitions de l'opinion citées dans la note 2. Mais dans son contenu elle attribue une importance ou une prépondérance (je vais expliquer cela), qui peut en partie éclairer la force extrême de l'impulsion qui en résulte. Regardons le texte suivant :

On pourrait objecter que la folie ne survient pas à cause de la faculté irrationnelle, mais parce que le jugement et l'opinion vont au-delà de ce qui est approprié, comme s'il [Chrysippe] voulait dire que les infirmités ( $\left.\dot{\alpha} \rho \rho \omega \tau \eta_{\mu} \mu \alpha \tau\right)$ émergent dans l'âme, non seulement de la conviction fausse que quelque chose est bon ou mauvais, mais de la conviction que cette chose est la plus grande [des biens ou des maux] ; l'opinion selon laquelle les richesses sont un bien n'est pas encore une infirmité, mais le devient quand on les considère comme le plus grand des biens ( $\mu \dot{\varepsilon} \gamma 1 \sigma \tau$ ov $\alpha \dot{\gamma} \alpha \theta$ òv) et que l'on suppose que la vie dépourvue de richesses ne vaut pas la peine d'être vécue : car c'est ce

10. Cf. Cicéron, Tusculanes, III, 61. Voir aussi Bénatouil 2009, p. 129-130, Donini 1995 et Tieleman 2003, p. 169.

11. Cf. Tieleman 2003, p. 252-253. 
en quoi consistent les infirmités que sont l'amour des richesses et l'amour de l'argent ${ }^{12}$.

Il faut également prendre en compte l'interprétation de Graver dans son ouvrage Stoicism and Emotions. Graver montre que, selon Chrysippe, la cause principale d'une passion est le caractère mental du sujet, où « caractère mental » signifie structure et contenu de l'ensemble des croyances qui sont propres à chacun ${ }^{13}$. Elle souligne que toutes les causes principales des passions sont de même type, à savoir des croyances. Sans ces croyances, il n'y aurait aucune réponse émotionnelle ${ }^{14}$. En ce qui concerne les critiques de Posidonius, Graver signale qu'elles peuvent être intégrées dans la théorie cognitive sans changements significatifs. L'approche de Chrysippe peut expliquer l'objection selon laquelle une croyance n'est pas suffisante pour produire une passion, en montrant l'importance des croyances supplémentaires qui ne figurent pas dans une première explication très simple ${ }^{15}$. Il s'agit donc de révéler les croyances qui fonctionnent dans chaque cas d'émotion et qui, parfois, ne sont pas évidentes.

Toutefois, le problème est que nous manquons de preuves textuelles, c'està-dire que nous ne disposons pas de textes qui abordent la difficulté directement et clairement. Je propose alors de l'analyser indirectement à travers des cas qui sont commentés dans la littérature que nous avons pu conserver, et qui illustrent par opposition le lien entre croyance faible et impulsion violente. Il s'agit du cas de Médée auquel Galien fait référence pour critiquer Chrysippe.

\section{Médée et le fil du temps : la théorie de la prépondérance des croyances}

Le cas de Médée, avec une si longue tradition dans la littérature grecque sur le conflit mental, est très significatif si nous pensons à la difficulté ici posée à la théorie cognitive ${ }^{16}$. Je ne vais pas présenter Médée comme un exemple d'akrasia, comme il est fréquent de le faire dans la littérature secondaire ${ }^{17}$. En effet, comme l'a montré Gourinat, il n'existe pas d'éléments suffisants permettant d'avancer que Chrysippe ait expliqué la colère de Médée en termes d'incontinence, ni que l'akrasia soit, en général, la source de toutes les

12. Galien, IV, 5, 24-26, p. 264-265 De Lacy. Je m'inspire de la traduction anglaise de De Lacy 2005 pour les lignes 24-25. Pour la dernière partie du texte, je cite la traduction de Brunschwig \& Pellegrin 2001 qui correspond au fragment LS 65L.

13. Cf. Graver 2007, p. 63-64.

14. Ibid. p. 66 avec référence aux Tusculanes, III, 24-25.

15. Cf. Graver 2007, p. 80.

16. Pour une analyse de la lecture que les penseurs antiques faisaient de Médée, voir Dillon 1997.

17. Voir Joyce 1995, Sorabji 2002, p. 227-228, et Tieleman 2003, p. 172 ; cités par Gourinat 2007, p. 242-246. 
passions ${ }^{18}$. Médée exhibe le conflit entre deux jugements, et la chose la plus intéressante dans son cas est qu'elle refuse de manière délibérée et consciente le jugement qui est en accord avec la droite raison. Ainsi, elle représente particulièrement bien la violence propre des passions ${ }^{19}$.

Dans sa délibération, Médée semble travailler avec au moins deux croyances contradictoires : une opinion faible et fausse, puisqu'elle attribue une grande valeur, dans ce cas-là négative, à un événement externe qui en réalité doit être indifférent, à savoir, l'offense de Jason, ce qui constitue le jugement de type 1 ; et un jugement de type 2.a, à savoir, « qu'il convient de se venger de cette offense en tuant mes enfants ${ }^{20}$, ce qui est aussi une opinion fausse et faible. Néanmoins, dans ce cas, et c'est là que se trouve la particularité, il y aurait, en agissant, un autre jugement que nous pourrions appeler jugement 2.b, à savoir, «qu'il ne faut pas se venger en tuant mes enfants $\gg^{21}$. Cette croyance est cohérente avec l'éthique stö̈cienne, car elle est fondée sur l'amour naturel de Médée pour ses enfants, et, donc, elle est correcte $^{22}$. Mais, dans le conflit qui se produit entre le jugement de type 2.a et

18. Les témoignages de Cicéron (Tusculanes, IV, 22-23), Galien (PHP, IV, 4, 24) et Sénèque (De Ira, II, 4, 1) ne peuvent pas justifier l'hypothèse contraire (cf. Gourinat 2007, p. 242-245; pour le cas de Médée, p. 245-246). Gourinat a insisté sur la complexité de la notion d'akrasia et d'enkrateia dans la philosophie stoïcienne. L'akrasia est un vice subordonné à l'akolasia, et l'enkrateia est une vertu subordonnée à la sophrosune, et toutes les deux, l'incontinence et la maîtrise de soi, se rapportent particulièrement au plaisir. Mais chez Cléanthe la maîtrise de soi est la vertu première, et même dans le cas de Chrysippe, il existe quelques textes qui illustrent la tendance à élargir le domaine de l'incontinence pour en faire le vice qui concerne les passions. Néanmoins, ce n'est pas la doctrine dominante ni chez Chrysippe ni dans le stoïcisme standard ( $c f$. Gourinat 2007, p. 246). L'incontinence n'est pas la source de toutes les passions. Par conséquent, il existe suffisamment d'arguments pour conclure que Chrysippe n'aurait pas appliqué la notion d'akrasia au cas de Médée.

19. Bénatouil a attiré l'attention sur le terme biastikon du texte de Stobée, Eclogae, II, 7, p. $89,4 \mathrm{~W}=$ SVF III, 378 et $389=$ LS 65A. Voir ci-dessus, n. 1. Ce terme est justifié, selon Bénatouil, par la référence à une expérience subjective de la passion, qui ne vaut plus pour toutes les passions, puisqu'elle arrive «souvent » (pollakis). Cette expérience est précisément celle du conflit intérieur. Cependant, ce n'est pas seulement la fréquence de cette expérience qui la rend pertinente pour comprendre la nature objective des passions, car, d'après Bénatouil, elle révèle la violence et la perte de contrôle qui définissent toutes les passions. Dans le conflit intérieur dont Médée est le paradigme, la contrainte et la division sont synchroniques et conscientes, donc manifestes subjectivement, alors que dans bien des passions, la désobéissance à la raison est inaperçue et l'emportement est tel qu'il n'est pas vécu comme une contrainte (Bénatouil 2009, p. 126-127).

20. Cf. Euripide, Médée, v. 1050-1055. Pour l'expression « il convient de/il faut agir ainsi », voir Bénatouil 2009, p. 129-130 avec référence aux Tusculanes, III, 61-62 et aux Lettres à Lucilius, 113, 18.

21. Cf. Euripide, Médée, v. 1040-1049 et 1056-1058.

22. L'amour pour les enfants est naturel et il n'existe pas chez les méchants. $C f$. Diogène Laërce, VII, 120. Il est très difficile de déterminer si le jugement 2.b de Médée est vrai ou faux, notamment face au jugement 2.a. En effet, le jugement 2.b n'exclut pas une autre croyance 
cet autre jugement 2.b, le jugement qui perd est précisément ce dernier : celui qui est vrai. En conséquence, l'impulsion excessive se déchaîne et Médée est poussée vers la vengeance. Il semble donc à nouveau que c'est l'opinion fausse et faible qui cause l'impulsion violente. Mais comment peut-on l'expliquer ? Comment une opinion fausse et faible peut-elle avoir un pouvoir causal plus grand qu'un jugement correct ?

Tout d'abord, il faudrait distinguer deux aspects du problème. Premièrement : pourquoi le faux l'emporte-t-il sur le vrai ? Dans ce cas, la solution s'avère plus facile car les stoïciens n'ont jamais dit qu'un jugement vrai avait en lui-même plus de force qu'un jugement faux. C'est seulement le jugement vrai fondé sur une représentation compréhensive qui est doté d'une force spéciale. Le jugement 2.b de Médée peut-il être de ce type, c'est-à-dire, un jugement vrai « kataleptique » ? Il semble clair que Médée n'est pas savante, car la science est absolument incompatible avec la passion pour les stoïciens. Cependant, selon le témoignage de Sextus Empiricus, les stoïciens affirment que la compréhension est commune aux sages et aux ignorants ${ }^{23}$. Par conséquent, il est possible que Médée ne soit pas savante mais qu'elle ait une compréhension. Ainsi, nous pouvons poser comme hypothèse que le jugement correct de Médée, le jugement 2.b, soit une katalepsis en raison de sa vérité, car l'opinion est particulièrement liée au faux ${ }^{24}$, et du fait de la ressemblance que l'exemple de Médée peut avoir, à première vue, avec un autre cas, celui de Ménélas et la représentation compréhensive qu' il a d'Hélène à la cour du roi Protée. Dans Hélène, la pièce d'Euripide, Ménélas ignore que, grâce à l'intervention d'Hermès, Pâris n'a enlevé qu'un fantôme qui ressemble à

fausse, à savoir se venger par d'autres moyens. Nous pouvons avancer que ce jugement est correct dans la mesure où il est fondé sur un amour naturel que l'on pourrait considérer «bon ». Néanmoins, le statut de l'amour n'est pas clair dans la philosophie stoïcienne. Il faudrait déterminer si l'amour peut ou non constituer une chose « indifférente préférable » que l'on pourrait appeler un bien ( $c f$. Plutarque, Des contradictions des stoïciens $1048 \mathrm{~A}=\mathrm{SVF}$ III, $137=\mathrm{LS}, 58 \mathrm{H}$ ) ou si l'amour peut être une eupatheia propre au sage ( $c f$. Ps. Andronicus, Depassionibus, 6 = SVF III, 432 ; voir aussi Graver 2007, p. 59). Toutefois, et malgré les difficultés, il me semble qu'il est intéressant et sensé de poser comme hypothèse que le jugement 2.b de Médée est vrai. En effet, cette hypothèse contient une valeur au moins heuristique, dans la mesure où elle permet de poser une autre question très pertinente pour éclaircir la nature du conflit de Médée, à savoir, si le jugement 2.b de Médée peut être un jugement « kataleptique $\gg$. La réponse - comme nous le montrerons ensuite - est négative. Le jugement 2.b est une opinion et, en ce sens, quelle que soit la valeur de vérité de cette opinion, elle est faible. La question de la vérité de cette croyance n'est pas déterminante et, soutenir qu'il s'agit d'une opinion fausse ne changerait pas le reste de l'argumentation que nous allons développer. Toutefois, il est pertinent de poser la question de la vérité afin, indirectement, de rendre le caractère de la lutte interne de Médée plus clair.

23. Cf. Sextus Empiricus, Adversus mathematicos, VII, 151-152. Voir aussi Cicéron, Seconds Académiques, I, 41-42b = SVF I $60=\mathrm{LS} 41 \mathrm{~B}$.

24. Cf. Diogène Laërce, VII 121 ; et Sextus Empiricus, Adversus mathematicos, VII 151 $=$ SVF I 67. 
Hélène et que la véritable Hélène se trouve en Égypte, chez Protée, depuis dix ans. Ménélas a des raisons bien fondées pour croire que Pâris a vraiment enlevé Hélène et que celle-ci est à Troie. C'est d'ailleurs ce qui a déclenché la guerre de Troie. En conséquence, lorsque Ménélas, qui revient de dix ans de guerre, rencontre la véritable Hélène à la cour du roi Protée, il n’en croit pas ses yeux (Hélène, v. 541-595) et il pense qu'il s'agit d'un fantôme. Ménélas ne donne pas son assentiment à la représentation compréhensive d'Hélène parce qu'il a des croyances bien ancrées qui fonctionnent comme obstacles ${ }^{25}$. Pour autant, aucun de ces arguments n'est suffisant pour justifier l'hypothèse selon laquelle le jugement 2.b de Médée est « kataleptique ». D’une part, il existe une deuxième définition stoïcienne de l'opinion selon laquelle l'opinion n'est qu'une conviction faible ${ }^{26}$, et donc, la fausseté n'est pas un attribut essentiel d'une opinion. Il peut y avoir des opinions vraies. Ainsi, le jugement 2.b de Médée peut être vrai sans pour autant cesser d'être une opinion ou une conviction faible, qui n'ait pas la fermeté de la katalepsis. D'autre part, le cas de Ménélas n'est pas complètement semblable à celui de Médée car l'idée qu'il ne faut pas tuer ses enfants n'est pas une évidence sensible à laquelle Médée a des raisons de ne pas croire. Le cas particulier de katalepsis qui s'oppose à un système de notions doit surtout s'appliquer à des évidences sensibles, et donc, il semble plus convenable de penser que le jugement approprié de Médée est une opinion vraie faible et non une katalepsis ferme ${ }^{27}$. Par conséquent, rien n'empêche une telle opinion de pouvoir être renversée par une autre, si cette dernière est prépondérante grâce à une raison ou une caractéristique qu'il faudrait - et c'est là la question - déchiffrer.

Ainsi, le problème ne se limite pas à éclaircir pourquoi le faux l'emporte sur le vrai, mais il inclut la question plus compliquée de la cause de la passion, à savoir, expliquer pourquoi l'opinion faible, sans la fermeté de la katalepsis, peut devenir si forte et emporter l'âme contre tous ses autres jugements. La difficulté principale consiste à élucider comment l'opinion faible peut l'emporter sur une opinion contraire et devenir une passion forte.

La réponse de Galien est facile et claire : la seule chose qui peut l'expliquer, c'est qu'il existe une autre force irrationnelle venant de la partie irrationnelle de l'âme, qui agit en faveur de l'impulsion émotionnelle, et qui entraîne sa violence. Le conflit dont souffre Médée est un conflit entre deux parties de l'âme différentes et opposées, à savoir, la partie rationnelle et la partie irrationnelle. Et c'est quelque chose que, selon Galien, Chrysippe aurait dû voir.

25. Cf. Sextus Empiricus, Adversus mathematicos, VII 154 et 256 ; voir Gourinat 1996, p. 75

26. Cf. Stobée, II, 111, 18-112, 8 = SVF III 548 = LS 41 G.

27. Je remercie Jean-Baptiste Gourinat de m'avoir éclairée sur ce point important par ses remarques très précises et claires. 
Mais si une personne est forcée sous l'emprise de la colère ou parce qu'elle est envoûtée par le plaisir de s'éloigner de son jugement initial, son âme est

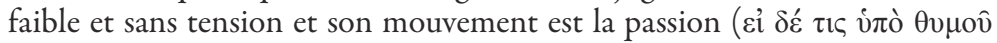

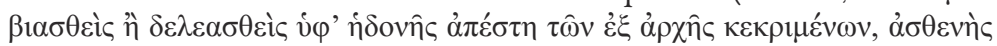

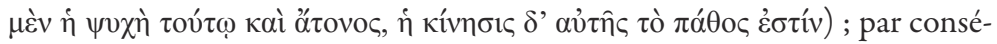
quent l'âme de Ménélas, tel que le présente la tragédie, renonce à sa décision car elle est envoûtée par le désir ; dans le cas de Médée, son âme est forcée par la colère ( nière, dans le cas de Médée, Chrysippe ne perçoit pas qu'il témoigne contre lui-même lorsqu'il cite les paroles d'Euripide :

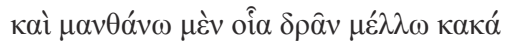

Je sais bien les maux que je vais faire,

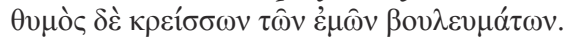

mais ma colère est plus forte que mes résolutions ${ }^{28}$.

Si Euripide avait voulu témoigner en faveur des enseignements de Chrysippe, il n'aurait pas dû dire que Médée comprenait, mais au contraire, qu'elle ignorait et qu'elle ne comprenait pas le mal qu'elle allait faire. Or avancer qu'elle le sait mais aussi qu'elle est emportée par la colère, qu'est-ce

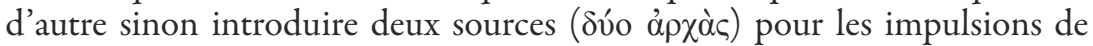

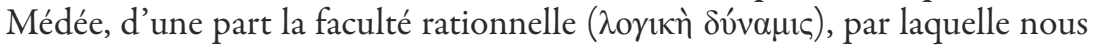
reconnaissons les choses et nous en avons connaissance, et d'autre part une

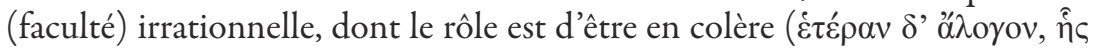

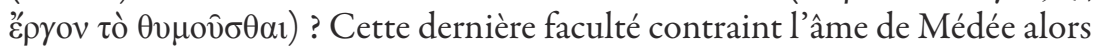

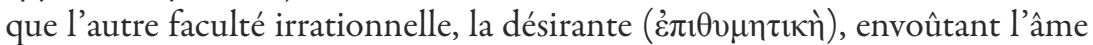
de Ménélas, l'oblige à suivre ses ordres. Comme certains sinterprètes l'ont fait remarquer, Chrysippe utilise l'exemple de Médée dans le but de différencier le type d'erreur propre à la passion, d'une erreur purement cognitive ou d'une erreur de raisonnement (hamartema) ${ }^{29}$. Les vers d'Euripide cités, « Je sais bien les maux que je vais faire, mais ma colère est plus forte que mes résolutions » (Médée, v. 1078-1079), montrent que Médée n'est pas aveugle. Le type d'erreur qu'Hercule, quand il est fou, commet lorsqu'il tue ses propres enfants en croyant que ce sont ceux d'Eurysthée, est comme

28. Galien, $P H P$, IV 6, 19-23, p. 274-275 De Lacy. Je m'inspire de la traduction anglaise de De Lacy 2005. Dans ce texte Galien semble faire référence aux vers 625-630 de l'Andromaque d'Euripide. Ménélas s'éloigne de son jugement initial de tuer Hélène après la guerre de Troie (voir la formulation dans Les Troyennes, v. 875) parce que son désir d'elle devient trop fort. En conséquence, il laisse tomber son épée par faiblesse à peine elle découvre son sein à sa vue (Andromaque, v. 629), d'où la comparaison avec Médée, que Galien souligne : la passion les empêche de faire ce qu'ils doivent faire. Les deux exemples montrent, selon Galien, qu'il existe une autre force venant de la partie irrationnelle de l'âme, qui produit la passion. Toutefois, comme je voudrais le montrer, la psychologie moniste mais non réductionniste de Chrysippe peut expliquer ces cas sans distinguer entre une partie rationnelle et une autre partie irrationnelle de l'âme.

29. Cf. Gill 1983 et Gourinat 1996. 
un assentiment donné à une fausse représentation ${ }^{30}$. En revanche, comme le précise Gourinat, Médée ne commet pas d'erreur de ce type. Médée sait que ce qu'elle va faire n'est pas bien, mais le désir de vengeance est plus fort, parce qu'elle a déjà donné son assentiment à la représentation de l'offense subie, et au fait que la vengeance est bonne. Elle s'est laissée aller à cette première représentation impulsive sans plus se rapporter à sa prénotion du bien. C'est en ce sens qu'elle tourne délibérément le dos à la raison ${ }^{31}$.

Galien est d'accord avec la thèse selon laquelle l'erreur caractéristique de la passion est différente d'une erreur purement cognitive. D'ailleurs, il se base sur cette distinction pour défendre sa thèse selon laquelle il doit exister une autre partie de l'âme différente de la partie rationnelle qui explique le problème de Médée, car la raison ne suffit pas pour expliquer son cas. En ce sens, Galien écrit en référence à Chrysippe :

De telles considérations montrent que la passion de l'âme ne survient pas dans le cas d'un objet inanimé ou d'un animal irrationnel. Et lorsqu'il écrit qu'un mouvement affectif survient en dehors de toute raison ou de tout jugement, il y ajoute ensuite les affirmations suivantes : «Ce n'est pas le cas lorsqu'une personne s'emporte par erreur et incompréhension de quelque chose qui est en accord avec la raison, et qu'elle agit également d'une manière qui rejette et désobéit à la raison ». Il distingue très clairement les passions et

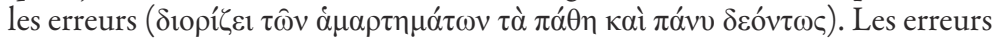

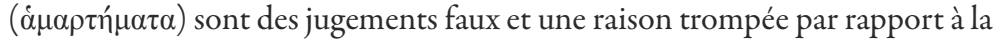
vérité et erronée. Alors que la passion n'est pas le fait de s'être trompé ou de commettre une erreur dans son raisonnement, c'est un mouvement de l'âme qui désobéit à la raison (

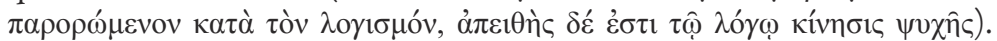
Un homme qui méprise la vie de ses enfants pour sauver son pays, que ce soit en les livrant à d'autres pour qu'ils les tuent ou en se chargeant lui-même d'accomplir l'acte, agit de cette façon parce qu'il lui semble noble de le faire, et il recourt à un certain raisonnement. Dans le cas de Médée, elle n'a été persuadée par aucun raisonnement pour tuer ses enfants, bien au contraire, pour autant qu'elle raisonne, elle dit qu'elle comprend que les actes qu'elle va commettre sont mauvais mais que la colère est plus forte que ses délibérations, c'est-à-dire que sa passion ne se soumet pas, n'obéit pas et ne suit pas la raison comme le ferait un maître, au contraire, elle s'en défait et s'éloigne des ordres et leur désobéit, ce qui implique que c'est l'action ou la passion d'une faculté autre que la faculté rationnelle. Comment une chose peut-elle se désobéir à elle-même, se rejeter elle-même, ou ne pas se suivre elle-même ${ }^{32}$ ?

Dans cette dernière partie du texte, il me semble que Galien est allé un

30. Sextus Empiricus, Adversus mathematicos, VII 405-407 ; cité par Gourinat 1996, p. 102.

31. Gourinat 1996, p. 101-102.

32. Galien, $P H P$, IV, 2, 23-27, p. 242-245 De Lacy. Je m' inspire de la traduction anglaise de De Lacy 2005. 
peu plus loin et qu'il ne suit pas le raisonnement de Chrysippe. Comme Gill l'a montré, Médée n'émet pas le type de jugement en accord avec la raison qu'une personne tout à fait rationnelle et raisonnable ferait. Mais, et c'est ce qui est frappant dans son cas, la réflexion de Médée implique un certain jugement, un certain exercice de la rationalité humaine, parce qu'elle comprend qu'elle va commettre un mal. D'ailleurs, comme l'avance Gill, Médée peut prendre conscience (rationnellement) qu'elle est irrationnelle. Le rejet et la désobéissance de la raison de Médée sont un rejet et une désobéissance conscients et délibérés. Il ne s'agit pas de la mise en échec de la raison par la passion, comme le veut Galien, mais d'un rejet délibéré de la raison par quelqu'un qui est suffisamment rationnel pour être conscient de ce qu'il est en train de rejeter. Chrysippe, d'après Gill, ne serait pas satisfait de l'interprétation de Galien selon laquelle Médée est entraînée alternativement par la passion et par la raison comme deux entités séparées et opposées ${ }^{33}$.

Toutefois, le problème est que Galien ne cite pas littéralement Chrysippe et qu'une telle citation n'existe pas dans les témoignages que nous avons conservés. Ainsi, il est difficile de savoir avec exactitude quelle est la réponse de Chrysippe à l'objection posée. Alors, en l'absence d'une citation littérale, je vais proposer une hypothèse, dans la lignée des travaux cités, et qui me semble plausible parce qu'elle est cohérente avec les textes qui ont été conservés (bien qu'il s'agisse d'une hypothèse probable qui peut être discutée). Le problème, encore une fois, consiste à expliquer pourquoi la raison est impuissante face à la raison même : comment peut-on expliquer que le jugement correct au sujet de la convenance d'un acte soit moins fort que la croyance fausse et faible selon laquelle il est bon de se venger, et, par conséquent, que cette croyance entraîne une impulsion violente ?

Je pense qu'il doit y avoir une autre caractéristique de la croyance faible, ou une autre croyance additionnelle qui n'est pas explicite en première approche, et qui cause l'impulsion excessive. C'est ce point que suggère la théorie de la prépondérance des croyances. Graver applique cette théorie de façon plus développée au cas d'Ulysse, dont Chrysippe parle également ${ }^{34}$. Dans ce cas, Ulysse n'affronte pas les prétendants de Pénélope, et l'impulsion émotionnelle n'entraîne pas une action. Des considérations d'intérêt propre, très pressantes, poussent Ulysse à rejeter l'action. D'après Graver,

33. Cf. Gill 1983, p. 140-142. Par ailleurs, le témoignage de Plutarque souligne qu' il n'y a pas d'opposition exclusive entre la raison et la passion : « Certains [les stoïciens] disent que la passion n'est pas autre chose que la raison et qu' il n'y a ni dissension ni conflit entre les deux, mais un infléchissement d'une raison unique dans deux directions, infléchissement que nous

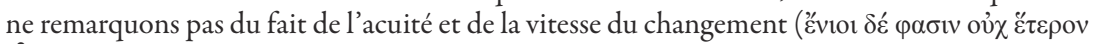

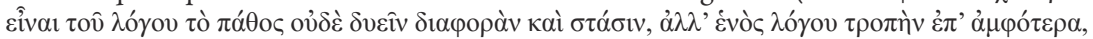

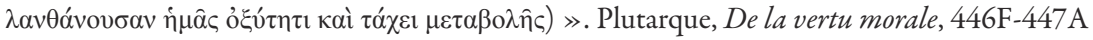
$=$ SVF III, $459=$ LS 65 G.

34. Cf. Galien, PHP, III, 3. 2-3. 
il convient de rappeler que, dans des situations spécifiques, le processus de délibération est extrêmement complexe, certaines croyances liées entre elles entrant en jeu ${ }^{35}$. Pour déchiffrer le cas d'Ulysse, Chrysippe aurait utilisé, d'après Graver, un modèle plus complexe, en avançant que, bien que l'impulsion émotionnelle dépasse généralement les considérations droites, il existe des cas où certains sujets importants (croyances), qui peuvent même ne pas être émotionnels, deviennent prioritaires ${ }^{36}$. Cette interprétation me semble très intéressante. Elle est liée à la théorie de la prépondérance des croyances de Nussbaum, à laquelle Graver fait référence. Â mon avis, cette théorie peut apporter une certaine solution à notre problème sans nous contraindre à renoncer au cœur de la théorie cognitive chez Chrysippe.

Nussbaum et Graver développent cette théorie pour répondre à une autre objection de Posidonius : comment est-il possible que la passion, et plus précisément le chagrin, perde de sa violence progressivement au fil du temps, voire disparaisse, et qu'en revanche le sentiment que quelque chose de mauvais est arrivé ne change pas $^{37}$ ? Nussbaum soutient qu'il existe au moins trois raisons pour lesquelles le chagrin peut diminuer avec le temps, bien que la croyance responsable du chagrin persiste. Il est possible que la croyance particulière ne se trouve pas au premier plan dans des situations concrètes, ou que d'autres croyances aient été ajustées en conséquence. $\mathrm{Ou}$ bien la croyance perd peut-être sa prépondérance parce qu'elle commence à être moins importante pour notre bonheur que d'autres croyances que nous entretenons ${ }^{38}$. C'est la raison que je trouve la plus intéressante par rapport à notre problématique. L'interprétation de Tieleman peut aussi répondre à cette objection. Il ne fait pas de référence explicite à la théorie de la prépondérance des croyances, mais sa réponse va dans le même sens. Tieleman signale qu'en réalité l'opinion change au fil du temps : non pas le jugement de type 1, « $\mathrm{X}$ est quelque chose de bon ou de mauvais », mais bien le jugement de type 2 sur la réponse appropriée à $\mathrm{X}^{39}$. Cette distinction entre le jugement 1 et le jugement 2 semble en accord avec la théorie de Nussbaum, car le fait qu'une opinion devienne moins importante pour l'agent, est en fait contenu dans le changement de jugement 2, par lequel l'agent estime qu'une réponse est convenable ou non, en fonction de ce qui le concerne actuellement.

$\mathrm{Si}$ nous appliquons maintenant la théorie de la prépondérance des croyances au cas de Médée, nous pouvons aboutir à la solution suivante : l'opinion 2.a selon laquelle il convient de se venger de Jason en tuant ses

35. Graver 2007, p. 71 fait référence à Sextus Empiricus, Adversus mathematicos, VII, 228-231.

36. Cf. Graver 2007, p. 71-72.

37. Cf. Galien, $P H P$, IV, 7. 4-7 = Posidonius, Fr. $165 \mathrm{EK}$.

38. Cf. Nussbaum 2001, p. 80-84.

39. Cf. Tieleman 2003, p. 138-139. 
enfants, acquiert une prépondérance majeure, c'est-à-dire qu'elle a plus de sens pour la perception de Médée, pour son épanouissement ou son bonheur personnel, que la croyance $2 . \mathrm{b}$, selon laquelle il ne convient pas de se venger de la sorte. La croyance 2. a fausse et faible est au premier plan par rapport au reste des croyances, compte tenu de la situation concrète que vit Médée et qui produit un réajustement de ses croyances. La prépondérance de l'opinion 2.a explique qu'elle l'emporte sur une autre croyance appropriée et qu'elle, l'opinion 2.a, cause une impulsion véhémente. La violence de l'impulsion correspond à l'importance de la croyance.

Mais, qu'est-ce qui explique la prépondérance de la croyance fausse et faible ? Graver, à propos de l'objection de l'affaiblissement de la passion au fil du temps, fait référence à l'argument de la « fraîcheur ». Les partisans de Chrysippe, d'après Graver, ne rencontreront pas de grand problème pour répondre à cette objection car la croyance qui provoque la passion, à savoir la croyance que « quelque chose de mauvais m'est arrivé » est équivalente à la croyance que « quelque chose de mauvais m'est arrivé récemment ». Le terme « récent » ou « frais » (prosphaton) qui apparaît dans la définition de Zénon, est très important. Selon Graver, cela ne veut pas dire que l'évènement doive réellement être récent, mais que l'agent doit le percevoir de cette façon. Il se pourrait d'ailleurs que des circonstances spéciales empêchent une personne de réaliser cet ajustement temporel, et que donc cette personne reste sous l'influence de la passion pendant très longtemps. Cicéron donne l'exemple de la peine incessante d'Artémise causée par la mort de son mari Mausole, roi de Carie. Sa désolation la conduisit à élever à Halicarnasse le célèbre Mausolée en l'honneur de son mari, et « subsista tant qu'elle vécut et d'ailleurs hâta sa fin en la minant lentement. Chez elle, l'idée du mal était tous les jours neuve, et pour que cette idée du mal ne soit plus qualifiée de neuve, il faut que le temps l'ait flétrie $\gg^{40}$. L'idée du mal - écrit Cicéron en faisant référence à Zénon et ses disciples - doit être qualifiée de neuve tant qu'il subsiste un élément qui lui donne de la vitalité et pour ainsi dire de la sève.

Peut-on éclairer le cas de Médée de cette façon ? Disons que nous pourrions introduire la variable du temps pour expliquer qu'une croyance fausse et faible produise une impulsion violente. Et il est possible, bien que cette interprétation s'éloigne des textes classiques mais pas tant que cela d'autres interprétations plus modernes de Médée, que le jugement approprié de type 2.b soit basé sur un bien que Médée voit aussi (la famille stable avec ses enfants) qui n'est pas certain et qui repose sur un avenir plus lointain, alors que le jugement de type 2.a ainsi que le jugement 1 invitent à une satisfaction immédiate. Le jugement 2.a répond dans l'instant à l'offense de Jason, très

40. Cicéron, Tusculanes, III, 75. Trad. J. Humbert (Fohlen 1931). Cf. Graver, 2007, p. $78-79$. 
proche dans le temps ; c'est pour cette raison qu'il acquiert la prépondérance suffisante pour entraîner une impulsion violente ${ }^{41}$. Toutefois, la mort de ses enfants est aussi une conséquence imminente sur laquelle Médée appuie son jugement 2.b. En effet, dans sa délibération, Médée fait référence au dernier sourire de ses enfants, c'est-à-dire, à leur mort, comme une raison pour abandonner son plan ${ }^{42}$. Par conséquent, la fraîcheur d'un évènement, ainsi perçu par le sujet, ne suffit pas pour expliquer la prépondérance du jugement 2.a, ni l'impulsion finale de Médée car tous deux font allusion à des faits récents.

Néanmoins, je pense que la théorie de la prépondérance des croyances peut aller plus loin sans se limiter à l'argument de la fraîcheur et qu'elle peut résoudre notre problème. L'argument de la fraîcheur n'est qu'une façon parmi tant d'autres d'expliquer la prépondérance d'une croyance. Cet argument pourrait effectivement convenir pour résoudre l'objection de l'affaiblissement de la passion au fil du temps, mais pas pour éclaircir notre difficulté. Mon intention est donc d'enrichir la théorie de la prépondérance avec un autre argument, tiré d'un témoignage de Posidonius, et que nous pouvons appeler l'argument de l'intensité de la valeur attribuée à l'événement. Dans ce texte, qui est rapporté par Galien, Posidonius refuse que l'élément de « fraîcheur » de la croyance puisse expliquer sa force pour causer une impulsion violente. Cependant, dans ce même texte, Posidonius indique implicitement une voie, fondée sur la même approche que celle de Chrysippe, pour résoudre notre problème. Ce texte est le suivant :

Il [Posidonius] demande qu'on lui dise ce qui fait que l'opinion d'un mal, lorsqu'elle est fraîche, contracte l'âme et provoque le chagrin, tandis que lorsqu'elle dure, elle ne provoque plus du tout cette contraction, ou du moins dans une moindre mesure. Or, si les enseignements de Chrysippe sont vrais, le mot « frais » ne devrait même pas figurer dans la définition. Il aurait été plus cohérent, compte tenu de son point de vue, de définir le chagrin comme l'opinion de la présence d'un mal considérable, ou intolérable, ou insoutenable, selon les expressions qui lui sont familières, plutôt que comme l'opinion d'un

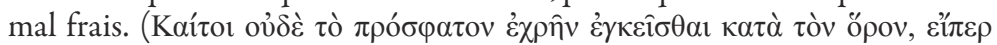

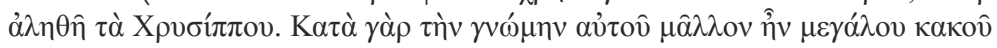

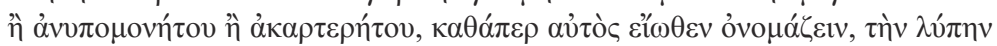

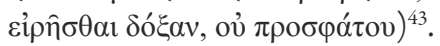

En ce qui concerne ce nouveau texte, il conviendrait d'éclaircir en premier lieu son contexte. Tout d'abord, cette constante insistance sur la cause de la cause, jusqu'à tomber presque dans une régression à l'infini, pourrait plus probablement être une stratégie de Galien que de Posidonius. Dans ce cas, il ne

41. Cf. Della Rocca 1996.

42. Cf. Euripide, Médée, v. 1040-1049.

43. Galien, $P H P$, IV, 7, 4-5, p. 280-283 De Lacy = Posidonius Fr. 165 EK. Je m' inspire de la traduction anglaise de De Lacy 2005. 
s'agirait pas d'une citation littérale de Posidonius. Et précisément, concernant l'objection du fil du temps, Galien ne semble pas agir très honnêtement. Et pour montrer ce qui n'est pas correct dans la théorie cognitive de Chrysippe, il écrit à la fin du livre IV: «Quel est celui qui, saturé avec le temps, change d'avis et rejette l'idée que deux fois deux font quatre ? Quel est celui qui rejette l'idée que tous les rayons d'un cercle sont égaux ? » ${ }^{44}$. Chrysippe a montré avec le cas de Médée que l'erreur de jugement qu'elle commet n'est pas la même erreur que celle de celui qui se trompe en additionnant deux plus deux. Même Galien le reconnaît devant Chrysippe. L'erreur même d'une passion n'est pas une erreur purement cognitive ${ }^{45}$. C'est une erreur morale, qui porte sur une représentation impulsive (phantasia hormetike) ${ }^{46}$, c'est-à-dire une représentation qui précède une horme. Cependant, l'erreur épistémique (deux fois deux font trois) porte sur une représentation qui n'entraîne aucune impulsion. Galien ne se rapporte pas ici aux jugements de valeur propres aux passions, mais à une autre sorte de jugements, car, de cette façon, son argument a une force majeure. Il s'agit à mon avis d'un argument certainement ad hominem et il montre le caractère inadéquat d'au moins une partie de la lecture que Galien fait de Chrysippe.

Toutefois, il me semble que le texte souligne un argument très intéressant pour notre propos, malgré la lecture de Galien. Je fais référence à l'idée selon laquelle Posidonius, d'après Galien, reconnaît un certain mérite à la proposition de Chrysippe qui consiste à ajouter une clause sur l'importance du bien et du mal attribuée à l'évènement en question. De cette façon nous pouvons lire : « si les enseignements de Chrysippe sont vrais ( $\varepsilon \grave{i} \pi \varepsilon \rho \dot{\alpha} \lambda \eta \theta \hat{\eta} \tau \grave{\alpha}$

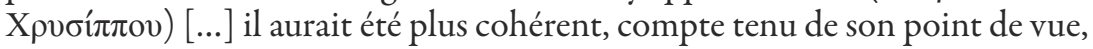
de définir le chagrin comme l'opinion de la présence d'un mal considérable, ou intolérable, ou insoutenable, selon les expressions qui lui sont familières,

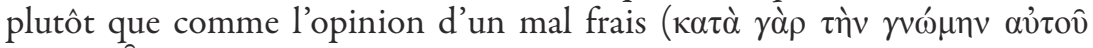

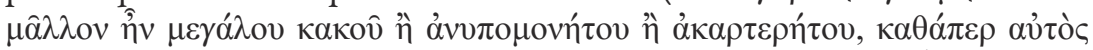

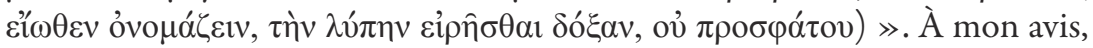
ce texte montre implicitement la pertinence de la théorie de la prépondérance des croyances par leur contenu, c'est-à-dire par le degré d'intensité du bien ou du mal attribué à l'événement. Il est vrai que dans le fragment cité ci-dessus, dans la deuxième section ${ }^{47}$, Posidonius rejetait l'idée que la cause d'une passion soit dans la grandeur du bien et du mal. En revanche, dans cet

44. Galien, PHP, IV, 7, 43. Trad. Fillion-Lahille 1984, p. 149.

45. Cf. Galien, PHP, IV, 2, 23-27. Voir supra, p. 166.

46. «Ce qui met en mouvement l'impulsion, disent-ils [les stö̈ciens], ce n'est rien d'autre qu' une impression capable d'impulser d'elle-même une fonction propre », Stobée, II, 86, 17 $=$ SVF III $169=$ LS 53Q.

47. Cf. Galien, PHP, IV, 5, 42-46, p. 269-270 De Lacy = Posidonius Fr 164 EK. Voir supra, p. 157-158. 
autre texte, qui est postérieur, le ton critique se relâche et Posidonius accorde un certain crédit et une certaine cohérence à l'approche de Chrysippe. De cette manière, la prépondérance de la croyance par son contenu acquiert une plus grande pertinence. Je pense par conséquent qu'il est possible d'expliquer le cas de Médée et la difficulté posée en développant la théorie cognitive de Chrysippe, et donc, en faisant référence aux croyances, à leur contenu et/ou aux rapports entre elles dans l'ensemble ou dans le système qui forme notre caractère $^{48}$.

Ainsi, cette clause à laquelle le texte fait allusion, à savoir la dimension du bien et du mal attribuée à l'objet, fait référence au contenu de la croyance ${ }^{49}$. La croyance, fausse et faible selon les stoïciens, que l'offense de Jason est un grand mal et qu'il convient de se venger en tuant ses enfants, est, d'un point de vue quantitatif, plus importante par rapport à l'idée que Médée a de son propre bonheur, que la croyance appropriée de la non-pertinence de cet acte. C'est la raison pour laquelle Médée lui donne son assentiment. Elle peut souscrire à la croyance selon laquelle sa vie sera malheureuse si elle ne se venge pas d'une manière comparable à la grandeur du mal infligé. En conséquence, la prépondérance de la croyance ne dérive pas de sa fraîcheur, mais du contenu de la croyance et de la manière dont Médée le voit.

Nous pouvons à présent voir plus clairement que l'opinion 1 exprime un grand mal, insoutenable pour Médée ${ }^{50}$, et que l'opinion 2.a apporte une réponse directe et proportionnelle à ce mal insupportable ${ }^{51}$. En effet, si Médée ne se vengeait pas en tuant ses enfants, elle serait la risée de ses ennemis, et cela serait pour elle, selon ses propres mots, insupportable. Ainsi Médée affirme :

48. C'est le seul point sur lequel je ne suis pas d'accord avec le texte, très intéressant et très bien fondé, de Gill 1998, p. 127-128, car il soutient qu'il n'est pas possible d'expliquer le cas de Médée par le contenu de la croyance ni par sa structure.

49. Si nous prenons en compte la thèse de Frede 1986, p. 103, la considération de l'offense de Jason comme un grand mal fait davantage référence à la manière dont Médée voit la proposition « l'offense de Jason s'est produite » qu'à la proposition même, de sorte que le jugement qui caractérise la colère de Médée est défini comme un assentiment à la représentation et non à la proposition. Cependant, comme l'a souligné Gourinat 1996, p. 70-71, il n'y a d'assentiment à donner qu'à des représentations qui ont une structure propositionnelle. De cette manière, on peut dire que la colère de Médée consiste à donner l'assentiment ou à juger vraie la représentation exprimée propositionnellement de la manière suivante : «l'offense de Jason est un grand mal ». Ce que je voudrais souligner est que la considération de l'offense comme un grand mal fait partie du contenu de la croyance qui définit la colère de Médée.

50. Cf. Médée, v. 225-230. Ici Médée affirme qu'elle veut mourir, qu'elle a perdu la joie de vivre car « celui qui pour moi était tout - je ne le sais que trop -, mon époux, est devenu le pire des hommes $\gg($ v. 228-229). La croyance 1 fait donc non seulement référence à une chose importante, mais aussi essentielle pour Médée.

51. Cette relation étroite entre l'opinion 2.a et l'opinion 1 se reflète à la fin de la tragédie lorsque Médée déclare que l'offense de Jason est la vraie cause de la mort de ses enfants (v. 1364). 
Après avoir ruiné toute la maison de Jason, je sortirai du pays, chassée par le meurtre de mes enfants bien-aimés, et l'abominable forfait que j'aurai osé. Être la risée de mes adversaires ! Non, je ne le supporterai pas, mes amies ${ }^{52}$ !

Bien que l'opinion 2.a fasse référence à une action impie, pour Médée, elle exprime finalement un bien, comme elle le reconnaît elle-même devant Jason à la fin de la tragédie. Ainsi en faisant référence au meurtre de ses enfants, Médée dit :

Oui. Mais ma douleur me sert, si tu n'as pas à rire ${ }^{53}$.

De cette manière, l'opinion 2.a fait référence à un grand bien, ou du moins elle exprime un bien plus grand pour Médée, que l'opinion 2.b fondée sur l'amour pour ses enfants. L'opinion 2.b ne repose pas sur une base très solide car, bien qu'il soit important, cet amour n'est pas essentiel pour Médée, contrairement aux moqueries de ses ennemis qu'elle doit absolument éviter, et qui constituent le fondement le plus fort de l'opinion 2. $a^{54}$. Même si Médée envisage la possibilité que ses enfants puissent faire sa joie (v. 1058), cela représente seulement une idée pour ainsi dire anecdotique. Il est évident qu'au début de la tragédie, lorsqu'elle parle de son histoire particulière, Médée ne fait pas référence à l'amour de ses enfants pour apaiser sa peine. À l'exception de cette petite évocation au vers 1058, elle ne mentionne plus par la suite cet amour pour calmer sa douleur. Elle cite plusieurs éléments qui pourraient rendre sa douleur moins forte ou plus supportable : avoir une cité, la maison d'un père, les avantages de la vie, etc. Mais elle ne fait aucune référence à (l'amour de) ses enfants parmi ces avantages de la vie.

Mais à toi et à moi ne sied pas le même langage. Ici tu as cité, demeure paternelle, avantages de vie et société d'amis. Moi, je suis seule, sans cité, en butte aux outrages d'un mari qui m'a ravie comme proie à une terre barbare, sans mère, sans frère, sans parent près de qui aller jeter l'ancre, loin de mon infortune $e^{55}$.

Nous pouvons donc conclure que l'opinion 2.a exprime un bien plus grand que l'opinion 2.b et et qu'elle offre une réponse comparable au mal insupportable que constitue, d'après Médée, l'offense de Jason (opinion 1). Par conséquent, grâce au degré du bien/mal qu'elle exprime ou avec laquelle elle est notamment liée, cette opinion occupe une place prépondérante dans l'ensemble des croyances de Médée. La théorie de la prépondérance peut expliquer, à partir de l'argument de l'intensité de la valeur, comment l'opinion faible 2.a peut l'emporter sur une opinion contraire (2.b) et devenir une passion forte.

52. Médée, v. 794-797.

53. Médée, v. 1362.

54. Il est frappant de constater la grande importance que Médée attribue à ce motif, attestée par les multiples fois où elle y fait référence tout au long de la tragédie.

55. Médée, v. 251-257. 
Par ailleurs, cette théorie souligne l'importance des rapports entre les croyances dans l'ensemble qui forme notre caractère mental. Nous disions au début de cette section que, comme l'explique Gourinat, « Médée s'est laissée aller à une première représentation impulsive de l'offense subie et de l'agrément de la vengeance sans plus se rapporter à sa prénotion du bien $\gg^{56}$. Médée n'établit pas une relation correcte entre ses jugements de valeur. La prépondérance de la croyance faible et fausse 2.a est telle qu'elle occupe toute son âme, de sorte que toutes ses représentations sont entièrement subordonnées à cette croyance. L'origine de la faute est, selon Gourinat, dans le fait de ne plus se rapporter qu'à une seule prénotion de ce qui lui est profitable dans la représentation de la situation présente, aux dépens de la prénotion du bien et du mal, au lieu de s'efforcer de rapporter cette représentation présente au système complet des notions et prénotions, comme à celle plus spécifique de son devoir de mère ${ }^{57}$. Médée a hiérarchisé incorrectement des croyances, elle les a ordonnées en un système analogue à une raison qui n'est pas droite, de sorte qu'elle a développé un caractère particulier. Cependant, comme nous venons de le voir, Médée mentionne son histoire particulière pour expliquer la gravité de son cas et cela peut induire en erreur. Il semblerait en effet qu'en dernier recours cette histoire, qui constitue l'expérience de Médée, explique une telle hiérarchie entre ses croyances et son action. En conséquence, est-ce la croyance ou l'expérience qui est la cause de la passion ? Il conviendrait d'éclaircir l'articulation entre ces facteurs.

\section{Croyance et expérience : vers un cognitivisme non réductionniste}

La vengeance a une capacité persuasive spéciale pour Médée. «Se venger » (opinion 1 et 2.a) a un impact singulier, étant donné l'histoire de Médée et son idiosyncrasie ${ }^{58}$. Son cas montre en partie comment 1) le pouvoir de persuasion des impressions et/ou 2) l'influence de la société, peuvent donner lieu à la corruption (diastrophe) du développement éthique naturel de l'être humain ${ }^{59}$. Sous l'influence de la société, nombre d'hommes

56. Gourinat 1996, p. 102.

57. Gourinat 1996, p. 105-106.

58. Le fait que la connexion entre une proposition et la force de l'impulsion soit une question idiosyncratique et relative au temps et à l'histoire de l'individu a été souligné par Joyce 1995, p. 326-327.

59. « Mais l'animal raisonnable est perverti, tantôt par les vraisemblances des réalités du monde extérieur, tantôt par l'influence de ceux qui partagent notre vie, puisque la nature fournit des points de départ exempts de toute perversion » (Diogène Laërce, VII, 89, trad. Goulet 2006). Bénatouil 2009, p. 131, parle de la même manière de la doctrine stoïcienne de la perversion de la raison et il conclut : « L'explication et la cure ultime du conflit intérieur de la raison réside ainsi plutôt dans l'épistémologie et la sociologie que dans la psychologie ». Cependant, cela ne signifie pas la perte du cognitivisme, car, comme je voudrais le montrer, il est toujours possible d'expliquer notre problème en faisant appel aux croyances. 
et de femmes peuvent accorder une grande importance à la réputation et estimer qu'ils doivent se venger lorsqu' ils sont trahis. Il est vrai que le cas de Médée est extrême et que, pour l'expliquer, il faut faire appel à la spécificité de son parcours, à ses expériences singulières ${ }^{60}$. Médée représente un risque extrême, mais ce risque, nous le courons tous dès que nous sommes séduits par les passions - et de ce fait nous pouvons comprendre pourquoi son cas a suscité tant d'intérêt chez les stoïciens. Le contexte social de Médée et les expériences particulières qu'elle a traversées tout au long de sa vie, et qu'ellemême évoque pour expliquer le caractère insupportable de l'offense de Jason, signalent implicitement des trahisons antérieures et les actes horribles que Médée a commis sous l'emprise des passions. La trahison et les actes impies ont un pouvoir de persuasion particulier pour Médée en raison de la familiarité qu'elle entretient avec eux.

Le contenu et l'agencement des croyances de Médée, qui définissent son caractère, sont un reflet de son expérience. Or ce sont les croyances mêmes, grâce à leur contenu et à leur organisation ou hiérarchie, qui sont la cause principale de sa passion. Il convient donc d'élucider comment les nombreux facteurs qui interviennent dans le cas de Médée peuvent être articulés selon l'approche de Chrysippe. En ce sens, il est fondamental de recourir à la distinction entre les causes que Chrysippe établit à propos de la question de la liberté de l'assentiment. Chez Cicéron nous pouvons lire :

Parmi les causes, dit-il [Chrysippe], certaines sont complètes et premières (perfectae et principales), d'autres auxiliaires et prochaines (adiuvantes et proximae $)^{61}$.

Les premières (perfectae et principales) sont les causes fondamentales des choses ou des actes, alors que les secondes (adiuvantes et proximae) constituent uniquement des facteurs causaux secondaires. Si nous appliquons cette distinction au cas de l'assentiment, la conclusion est la suivante :

De même que celui qui a poussé le cylindre lui a donné le commencement de son mouvement, mais non sa capacité de rouler, de même, bien que l'impression que l'on rencontre imprime, et, pour ainsi dire, grave son apparence dans l'esprit, l'assentiment sera en notre pouvoir. Et l'assentiment, comme dans le cas du cylindre, bien que provoqué du dehors, se mouvra par la suite selon sa propre force et sa propre nature ${ }^{62}$.

C'est le contenu et le rapport entre les croyances qui déchaînent la colère de Médée, bien que ce qui cause la formation de la croyance soit le contexte social ou la capacité persuasive des impressions.

60. Pour l'importance de l'expérience et de l'enseignement chez les stoïciens, voir Aétius IV, $11,1-4=$ SVF II, $83=$ LS 39E.

61. Cicéron, Du destin, 39-43 = SVF II, $974=$ LS 62C.

62. Du destin, 39-43. Il convient de noter que Chrysippe revient à l'exemple du cylindre, qui est aussi un lieu commun dans le contexte de notre problématique. 
De même que la cause principale de l'assentiment ou du mouvement du cylindre est leur propre force et leur propre nature, de même la cause principale de la passion de Médée est le contenu et la hiérarchisation de ses croyances dans l'ensemble qui forme son caractère mental, même si le concours de facteurs causaux secondaires, comme les expériences particulières que Médée traverse dans son contexte social, de la même façon que la poussée dans l'exemple du cylindre, est nécessaire pour que l'action se déclenche effectivement, ou en d'autres termes, pour que le caractère mental s'exerce effectivement comme une cause. Ce qui est plus difficile à déterminer, c'est si le caractère mental est une cause complète ou première, c'està-dire s'il constitue une cause suffisante ou non. Il me semble qu'il est plus vraisemblable qu'il représente une cause première si nous utilisons l'analogie du cylindre pour comprendre ce problème, car cette analogie montre que la poussée est nécessaire pour que le mouvement du cylindre se déclenche effectivement. Les causes premières, comme l'a si bien montré Sedley, sont celles qui ont une plus grande responsabilité pour l'effet sans être suffisantes, car certaines conditions appropriées doivent avoir lieu pour que l'action se produise $^{63}$. Ces conditions pourraient être les expériences particulières de Médée dans la société de son temps. Or la cause première de sa passion est son caractère mental, c'est-à-dire, l'ensemble de ses croyances. Quoi qu'il en soit, qu'il constitue la cause complète ou première, il me semble clair que, si nous suivons la distinction fondamentale qu'établit le texte cité, le caractère mental appartient aux causes de type 1 ou aux causes fondamentales, et l'expérience (l'influence de la société, etc.) appartient aux causes de type 2 ou causes seulement secondaires.

Nous pouvons à présent mieux comprendre non seulement comment les facteurs causaux se rejoignent pour expliquer le cas de Médée, mais aussi comment les diverses théories évoquées (prépondérance des croyances, thèses sociologique, physique etc.) s'articulent, et en quoi cette articulation est cohérente avec l'approche cognitive de Chrysippe. Il convient de distinguer à cet égard deux niveaux d'analyse : l'un, pour ainsi dire, microscopique, qui concerne le point de vue du contenu d'une croyance déterminée ; et l'autre, macroscopique, qui concerne le point de vue psychologique général. Dans un sens plus précis, notre problème concerne une question plus microscopique ou concrète : comment est-il possible qu'une opinion faible puisse emporter l'âme contre tous ses autres jugements et devenir ainsi une passion forte ? À ce niveau de l'analyse, nous pouvons appliquer la théorie de la prépondérance des croyances pour résoudre la difficulté. La réponse que nous avons trouvée

63. Cf. Sedley 1993 , p. 322-324. Sedley penche pour la thèse selon laquelle le caractère moral du sujet est une cause première et non complète et il élucide avec beaucoup de détails les caractéristiques de chacune. Je renvoie à son intéressant travail auquel je fais référence de manière implicite dans mon argumentation. 
à cet égard consiste à affirmer que c'est grâce à l'intensité de la valeur que cette opinion exprime (insupportable, considérable ou insoutenable) qu'une telle opinion peut devenir si forte et causer une impulsion violente. Cette opinion concerne non seulement le bien ou le mal, mais également les choses essentielles à notre bonheur. Elle concerne ce qui est important, mais aussi ce qui est essentiel, et de ce fait elle se positionne en haut de notre hiérarchie des croyances. La théorie de la prépondérance des croyances, de par son contenu, permet d'expliquer notre problème d'un point de vue cognitif. C'est le contenu de la croyance qui explique qu'une opinion faible puisse se traduire par une impulsion violente.

Cependant, au niveau macroscopique, les facteurs qui interviennent dans le déclenchement de l'action de Médée sont, d'après le stoïcisme, multiples, bien qu'ils n'aient pas le même degré de responsabilité, et la détermination sociale ou personnelle de l'individu s'avère être un second facteur décisif. Nous venons de voir comment l'expérience de Médée pose les conditions appropriées pour l'action. Néanmoins, le cognitivisme ne disparait pas à ce niveau dans la mesure où c'est le caractère mental ou l'ensemble des croyances de Médée qui constituent la cause fondamentale de son impulsion débridée ou de sa passion. L'explication cognitive peut être complétée par l'approche sociale ou individuelle, même si elle est d'une certaine manière supérieure. La réponse à notre problématique est par conséquent de nature cognitive pour les deux niveaux d'approche, mais de façon non réductionniste.

Enfin, l'explication physique à laquelle nous avons fait référence dans notre commentaire sur l'interprétation de Tieleman trouve sa place ici tout autant. Quel que soit le niveau d'analyse envisagé, celui du contenu déterminé de la croyance ou de la psychologie plus large, l'explication physique constitue toujours la contrepartie de l'explication cognitive, c'est l'autre face de la pièce de monnaie pour ainsi dire. Chez Chrysippe, le dualisme n'existe pas. Le corps n'est pas une cause plus importante que le caractère mental, ou vice versa, comme s'il s'agissait de deux entités opposées. Au contraire, ils constituent tous les deux, dans un certain sens, les deux faces d'une même chose. L'esprit stoïcien est, comme le souligne Sedley ${ }^{64}$, emphatiquement corporel ou encore, selon Tieleman, dans la psychologie de Chrysippe, la croyance et l'aspect physique ne sont pas incompatibles mais ils s'impliquent mutuellement. 


\section{Conclusion}

La théorie cognitive des passions chez Chrysippe peut-elle résoudre le problème posé ? Pour y répondre, j'ai essayé tout au long de cet article de développer la théorie de la prépondérance des croyances de Nussbaum et de Graver en m'appuyant sur ce que j'ai dénommé l'argument de la magnitude/ intensité de la valeur, à partir d'un témoignage de Posidonius sur la philosophie de Chrysippe. Ainsi, cette théorie dans sa version enrichie peut expliquer, à partir de la grandeur de la valeur que la croyance faible exprime pour le sujet, pourquoi une opinion comme celle de Médée peut devenir une passion forte ou déchaîner une impulsion débridée. La prépondérance, comme nous venons de le montrer, est liée au contenu de la croyance, c'està-dire à la dimension du bien et du mal qu'elle exprime. Elle met par ailleurs en évidence l'importance des rapports entre les croyances qui forment notre caractère mental, car la hiérarchisation incorrecte des opinions chez Médée explique aussi son action. La théorie cognitive des passions peut donc effectivement éclairer la difficulté par une certaine caractéristique de la croyance, sans besoin de faire appel à une autre partie irrationnelle de l'âme comme le veut Galien. Je pense que la théorie cognitive des passions de Chrysippe aurait pu aller dans cette direction, car le développement du cognitivisme que nous avons formulé pourrait expliciter certains aspects implicites dans sa philosophie.

Par ailleurs, la théorie cognitive de Chrysippe est suffisamment flexible pour admettre que d'autres facteurs sont impliqués dans l'analyse du problème. Toutefois, elle souligne que ces facteurs n'ont pas le même type ou le même degré de responsabilité. Les facteurs de caractère social ou idiosyncrasique sont secondaires par rapport au rôle causal de premier rang que jouent nos croyances. Quant à l'explication physique, elle est complémentaire et non contradictoire avec l'analyse en termes de croyances. Nous pouvons soutenir que les passions sont des croyances, des opinions ou des jugements, mais nous ne pouvons pas nier qu'il existe une dimension corporelle correspondante. Le développement éthique déficient de nos croyances correspond à un manque de force (atonia), dont fait preuve Ménélas lorsqu'il laisse tomber l'épée ${ }^{65}$. Le dualisme n'existe pas dans l'approche de Chrysippe. Ainsi, le développement que nous avons proposé ici de sa théorie étaie le noyau du cognitivisme, sans pour autant que ce dernier soit réductionniste. Je pense que Chrysippe, bien que ce point ait été perfectionné par les stoïciens ultérieurs, admet que la recherche sur les passions doit être ductile. C'est peut être là le chemin à suivre pour résoudre ou, tout au moins, pour atténuer les difficultés que rencontre la théorie cognitive des passions dans sa philosophie. 


\section{BIBLIOGRAPHIE}

Arnim, H. von 1903-1924 (éd.) : Stoicorum veterum fragmenta (= SVF), I, Zeno et Zenonis discipuli ; II, Chrysippi fragmenta logica et physica; III, Chrysippi fragmenta moralia : Fragmenta successorum Chrysippi; IV, Quo indices continentur conscripsit Maximilianus Adler, Leipzig, 1903-1924.

BÉNATOUÏL, T. 2009 : « La raison stoïcienne face à elle-même : le statut du conflit intérieur dans la psychologie morale de Chrysippe », dans R. Lefebvre \& A. Tordesillas (éd.), Faiblesse de la volonté et maîtrise de soi : doctrines antiques, perspectives contemporaines, Rennes, 2009 (Philosophica), p. 119-131.

Brunschwig, J. \& P. Pellegrin 2001 (trad.) : A. A. Long \& D. N. Sedley, Les philosophes hellénistiques, 1, Pyrrhon. L'épicurisme; 2, Les stö̈ciens; 3, Les académiciens : la renaissance du pyrrhonisme, textes traduits par J. Brunschwig \& P. Pellegrin, Paris, 2001 (GF Flammarion, 641-642-643).

Cooper, J. M. 1998 : « Posidonius on Emotions » dans J. Sihvola \& T. EngbergPedersen 1998 (éd.), p. 71-112.

De Lacy, Ph. 2005 (éd.) : Galen, De Placitis Hippocratis et Platonis - On the doctrines of Hippocrates and Plato, 1, Books I-V; 2, Books VI-IX ; 3, Commentary and indexes, Berlin, 2005 (Corpus Medicorum Graecorum V, 4, 1, 2).

Della Rocca, M. 1996 : « Spinoza's Metaphysical Psychology », dans D. Garret (éd.), The Cambridge Companion to Spinoza, Cambridge, 1996 (Cambridge Companions to Philosophy), p. 167-258.

Dillon, J. 1997 : « Medea among the Philosophers » dans J. J. Clauss \& S. I. Johnston (éd.), Medea : essays on Medea in Myth, Literature, Philosophy and Art, Princeton, 1997 (Princeton paperbacks), p. 211-218.

Donini, P. 1995 : « Struttura delle passioni e del vizio e loro cura in Crisippo », Elenchos: Rivista di studi sul pensiero antico, 16/2 (1995), p. 305-329.

Dumortier, J. 1975 (éd.) : Plutarque, CEuvres morales, 7, 1, Traités 27-36, texte établi et traduit, avec la collaboration de J. Defradas, Paris, 1975 (Collection des Universités de France. Série grecque, 236).

Edelstein, L. \& I. G. Kidd 2004-2005 (éd.) : Posidonius, The fragments, 1, The fragments ; 2, The commentary; 3, The translation of the fragments, Cambridge, 2004-2005 (Cambridge Classical Texts and Commentaries, 13, 14A, 14B, 36).

Fillion-Lahille, J. 1984 : Le De ira de Sénéque et la philosophie stoücienne des passions, Paris, 1984 (Études et commentaires, 94).

Fohlen, G. 1931 (éd.) : Cicéron, Tusculanes, t. 2, Livres III-V, traduit par J. Humbert, Paris, 1931 (Collection des Universités de France. Série latine, 65).

Frede, M. 1986 : « The Stoic Doctrine of the Affections of the Soul », dans M. Schofield \& G. Striker (éd.), The Norms of Nature : Studies in Hellenistic Ethics, Cambridge-Paris, 1986, p. 93-110.

GILl, C. 1983 : « Did Chrysippus understand Medea? », Phronesis, 28/2 (1983), p. 136-149.

-1998: «Did Galen Understand Platonic and Stoic Thinking on Emotions ? », dans J. Sihvola \& T. Engberg-Pedersen 1998 (éd.), p. 113-148.

Goulet, R. 2006 (trad.) : Diogène Laërce, Vies et doctrines des Stoüciens, Paris, 2006 (Classiques de la philosophie). 
Gourinat, J.-B. 1996 : Les stö̈ciens et l’âme, Paris, 1996 (Philosophies, 75).

-2007 : « Akrasia and Enkrateia in Ancient Stoicism : Minor Vice and Minor Virtue ? », dans C. Bobonich \& P. Destrée (éd.), Akrasia in Greek Philosophy: from Socrates to Plotinus, Leiden, 2007 (Philosophia antiqua, 106), p. 215-247.

Graver, M. 2007 : Stoicism \& Emotion, Chicago-London, 2007.

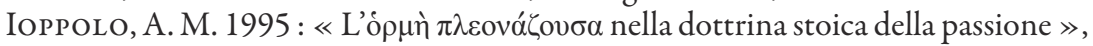
Elenchos. Rivista di studi sul pensiero antico, 16 / 1 (1995), p. 23-55.

Joyce, R. 1995 : « Early Stoicim and Akrasia », Phronesis, 40/3 (1995), p. 315-335.

KIdD, I. G. 1971 : « Posidonius on Emotions », dans A. A. Long (éd.), Problems in Stoicism, London, 1971, p. 200-215.

Long, A. A. \& D. N. Sedley 1987 (éd.) : The Hellenistic Philosophers, 1, translations of the principal sources, with philosophical commentary; 2, Greek and Latin texts with notes and bibliography, Cambridge, 1987.

Méridier, L. 1925 (éd.) : Euripide, Tragédies, 1 : Le Cyclope, Alceste, Médée, Les Héraclides, Paris, 1925 (Collection des Universités de France. Série grecque, 37).

Nussbaum, M. C. 2001 : Upheavals of Thought : the Intelligence of Emotions, Cambridge, 2001.

Pomeroy, A. J. 1999 (éd.) : Arius Didymus, Epitome of Stoic Ethics, Atlanta, 1999 (Texts and Translations, 44. Graeco-Roman series, 14).

Sedley, D. 1993 : « Chrysippus on psychophysical causality », dans J. Brunschwig \& M. C. Nussbaum (éd.), Passions and Perceptions : Studies in Hellenistic Philosophy of Mind: proceedings of the Fifth Symposium Hellenisticum, Cambridge, 1993, p. 313-331.

Sinvola, J. \& T. Engberg-Pedersen 1998 (éd.) : The Emotions in Hellenistic Philosophy, Dordrecht, 1998 (The New Synthese Historical Library, 46).

SorABJI, R. 2000 : Emotion and Peace of Mind: from Stoic agitation to Christian temptation, Oxford, 2000 (The Gifford Lectures).

-2002: «Zeno and Chrysippus on Emotions », dans Th. Scaltsas \& A. S. Mason (éd.), The Philosophy of Zeno : Zeno of Citium and his Legacy, Larnaca, 2002, p. 221-238.

Tieleman, T. 2003 : Chrysippus' On Affections : reconstruction and interpretation, Leiden, 2003 (Philosophia antiqua, 94). 